\title{
Efficient Control System Based on Hand Nerve Signals
}

\author{
Ahmed Majid Abdel Abbas ${ }^{1}$, Muayad Sadik Croock ${ }^{1}$ \\ Computer Engineering Department, University of Technology-Iraq, Baghdad Iraq \\ ahmedalthahabi1993@yahoo.com,120102@uothecnology.edu.iq
}

\begin{abstract}
Recent advances in the control applications based on hand nerve signals are able to meet the needs of users who suffer from restrictions in limb movement and also provide high performance control for those paralyzed people. These signals are represented as Electromyography (EMG) signals, which are biomedical ones, used for clinical/biomedical applications. In this work, a control signals generation system is proposed based on hand EMG measurements. The process of acquisition and processing of EMG signals is performed by only one channel surface EMG electrodes with one EMG processing unit as a muscle sensor. In this work, Arduino UNO is adopted as an analog to digital converter for these hand nerve signals to be easily analyzed in the classification process. These signals are measured from the skin surface of forearm and biceps muscles in two suggested case studies to be used in generating signals based on ten muscles movements. The main features that crystallized this research is building a smart control algorithm which increases the flexibility of generating precise control signals based on contracted hand movements with high simplicity of use and the low cost. The obtained results are compared to other systems results to show the ability of achieving $93.81 \%$ classification rate or accuracy among other systems.
\end{abstract}

Index Terms-Arduino UNO, Biomedical application, Control signals, Electromyography.

\section{INTRODUCTION}

Upper-limb motions, which can be characterized and estimated using the activation of the upper-limb muscles, are very important for many daily human activities but the loss of the human upper limb is a major disability that terribly limits the everyday capabilities and interactions of individuals and it is difficult for physically amputated and injured individuals to perform those activities [1]. Moreover, it also leads to depression along with other psychological risks. Usually, most frequent causes of upper limb amputation are trauma and cancer, followed by vascular complications of disease of right arm that is more frequently involved in work [2].

The EMG based control system is considered the best utilized system recently when compared with other systems due to its non-invasive behavior [3]. EMG-based control system can be separated into two main groups: Pattern recognition and non-pattern recognition. In pattern recognition-based EMG systems, a classifier such as SVM, ANN, LDA, etc, are adopted to classify signal patterns as control commands that are extracted from raw data in the form of features which are extracted and classified to generate the control commands. The non-pattern recognition is focused on the thresholding or onset analysis method, using finite state machine. However, the main limitation of non-pattern recognition method is that it restricts the number of control commands [4]. In general, this system belongs to the non-pattern recognition EMG based systems because of the fact that the control signals that are generated are few. 
The aim of this paper is to propose an affordable and flexible control system based on hand nerve and EMG readings of amputees people. The proposed system utilizes one channel surface EMG (sEMG) electrodes with only one MyoWare muscle sensor and Arduino UNO microcontroller as an analog to digital converter. This is for generating ten control signals according to muscles movements for two case studies, considered for processing different hand amputation cases. These cases include people who suffer from wrist disarticulation (through the wrist joint), fingers, the palm of the hand amputation, and forearm of the hand (through the hand attachment point). The generated control signals can be used for controlling robotics, moving objects and other devices according to the designed suggested algorithm. The proposed system is tested amongst the adopted two case studies, each one with five muscle movements. The obtained results show a classification accuracy of almost $93.81 \%$.

\section{RELATED WORKS}

Control applications of EMG signals had become a crucial part of researches in recent years, especially under the recent enormous increase of the elderly and disabled people. Those researchers adopted numerous studies based on hand nerve signals for helping disabled people in different sides.

In [6], the researchers proposed a novel method to estimate the finger motions using surface EMG signals. This proposed system employed four EMG channels placed around the forearm for classifying eight fingers motions. The result was positive but the system was costly and it restricted the movement of the amputees. In [7], the authors developed a lower arm prosthesis based on EMG signals for classifying seven different movements by using three channels with a bipolar configuration, six surface electrodes plus only one another electrode as a reference that were located beside the forearm muscles. Morever, the proposed system offers a high classification accuracy by employing the motions of hand fingers instead of the wrist in order to enhance the performance of prosthesis by doing complex tasks, but the real time response was slower according to the ANN classifier used. In [8], the researchers suggested a system for classifying finger movements of the upper limb prosthesis based on EMG signals for helping disabled people to bring those people back to normal life. The proposed system used twelve EMG channels for 8 classes of independent finger movements plus a rest class i.e. flexing of little, ring, middle, and index fingers and rest. With extension of little, ring, middle, and index fingers and the rest of fingers which are considered a class in this system. The classification rate of movements was positive but the system was very costly. In [9], the researchers designed a system toward improved control of prosthetic fingers using sEMG signals for classifying ten individual and combined fingers. This was done using only two EMG channels placed on the forearm of the human. This project uses LIBSVM (LIBrary for Support Vector Machine) and KNN (k Nearest Neighbor) as classification techniques. The classification result with LIBSVM could provide lower error rate when compared to that of KNN classifier but it took more time and hence the classification accuracy of this system was roughly equal $90 \%$. The positive point of this system was its medium cost and simplicity but the computational cost needed more time to output the control motion. The authors of [11], published a paper to evalute the gestures of the hand based on the classification of EMG signals. the proposed acquisition system employs two channels of surface EMG for classifying seven motion classes, which made the system simple and has the lowest cost. However, all proposed gestures require three seconds to be performed with another three seconds for the rest action that were placed between gestures, which reduced the response time.This project employed seven classifiers but support vector machine (svm) classifier required more time during the EMG classification stage. In [14], the authors evaluated the different approches of feature extraction and classifiers for fingers actions based on EMG signals. This system classified fourteen finger movements that were acquired by six channels with employing six methods of feature extraction and seven classifiers. This system had accomplished 99\% classification accuracy 
according to SRELM and NN classifiers in combination. This system was very costly and it restricted the movement of the paralyzed people.

Moreover, this work has highlighted additional research regarding this field and all of these researches are based on EMG signals as control signals. These researches are summarized in Table I.

TABLE I. CONTROL APPLICATIONS OF PREVIOUS RESEARCHES BASED ON EMG SIGNALS.

\begin{tabular}{|c|c|c|c|c|}
\hline Researches & $\begin{array}{c}\text { No. of } \\
\text { EMG } \\
\text { Channels }\end{array}$ & $\begin{array}{c}\text { Electrodes } \\
\text { Location }\end{array}$ & $\begin{array}{c}\text { No. of } \\
\text { Control } \\
\text { Commands }\end{array}$ & $\begin{array}{c}\text { Average } \\
\text { Classification } \\
\text { Accuracy }\end{array}$ \\
\hline Tsenov, G., et al [5] & two & $\begin{array}{c}\text { Forearm } \\
\text { muscles }\end{array}$ & 4 & $92.64 \%$ \\
\hline $\begin{array}{c}\text { Anam, K., Khushaba, R. N., } \\
\text { \& Al-Jumaily, A. [10] }\end{array}$ & two & $\begin{array}{c}\text { Forearm } \\
\text { muscles }\end{array}$ & 10 & $>90 \%$ \\
\hline $\begin{array}{c}\text { Puttasakul, T., Sangworasil, } \\
\text { M., \& Matsuura, T. [12] }\end{array}$ & four & $\begin{array}{c}\text { Arm } \\
\text { muscles }\end{array}$ & $61.6 \%$ \\
\hline $\begin{array}{c}\text { Gregory Luppescu, Michael } \\
\text { Lowney \& Raj Shah [13] }\end{array}$ & ten & $\begin{array}{c}\text { Arm } \\
\text { muscles }\end{array}$ & 6 & $93 \%$ \\
\hline
\end{tabular}

\section{METHODOLOGY}

In humans, all the accomplished movements from limbs are generated due to the contractions of body muscles, in the fact that many electrical signals are reached to the desired muscles by the nervous system through the motor neurons.

The EMG signal is a biomedical signal that represents the electrical articulation of the offering neuromuscular activities related with shrinking of muscles [15]-[17]. However, these EMG signals are stochastic in nature and can be represented by a Gaussian distribution function. The amplitude of these signals is very small and can be ranged from 0 to 10 millivolt (peak-to-peak) or 0 to 5 millivolt (rms). The usable energy of the signal is limited to a specific frequency range of (0 to $500 \mathrm{~Hz})$ [18].

In this work, one channel surface EMG (sEMG) electrodes with only one EMG processing unit as a muscle sensor have been adopted for processing these EMG signals and thus the output signals from myoware muscle sensor are amplified, rectified and filtered to be easily interfaced with any analog to digital converter such as the arduino microcontroller. These signals generate ten control commands according to ten hand motions. Surface electrodes are used to record the raw EMG signals from predefined muscles but the placement of these surface electrodes on the target muscles groups is very vital to record more precise and proper information of upper limb actions. EMG signals are acquired by means of one channel in bipolar setting (two electrodes plus one reference electrode) which are placed on the forearm and biceps muscles in two case studies in this study. The electrodes send the acquired hand nerve signals to EMG processing unit such as Myoware muscle sensor for denoising and strengthening these weak signals.

\section{The PROPOSED SYSTEM}

In general, the structure of the proposed system involves four main parts. Each of which has a function for completing the required job in an acceptable accuracy and efficiency.

\section{A. Hardware Equipment}

The proposed system consists of simple electronic and electric components including muscle sensor, microcontroller, bread board, jumper wires, LEDs, and two of (9 volt) batteries. This system mainly focuses on the Myoware muscle sensor.

Myoware muscle sensor (AT-04-001), as illustrates in Figure (2.17), is an electromyography sensor especially designed for control applications [19], and it was developed by Advancer Technologies in 
United States to be adopted for acquiring EMG signals and its elaboration in several areas by measuring the activity of muscles fibers via electric potential that is recorded by surface electrodes and transforming this into variable electrical voltage that can be read by any analog input pin of any microcontroller, and therefore this sensor is designed to be compatible directly with any ADC microcontrollers, such as an A-Star and Arduino [20]. Fig. 1 shows the MyoWare muscle sensor structure [21].

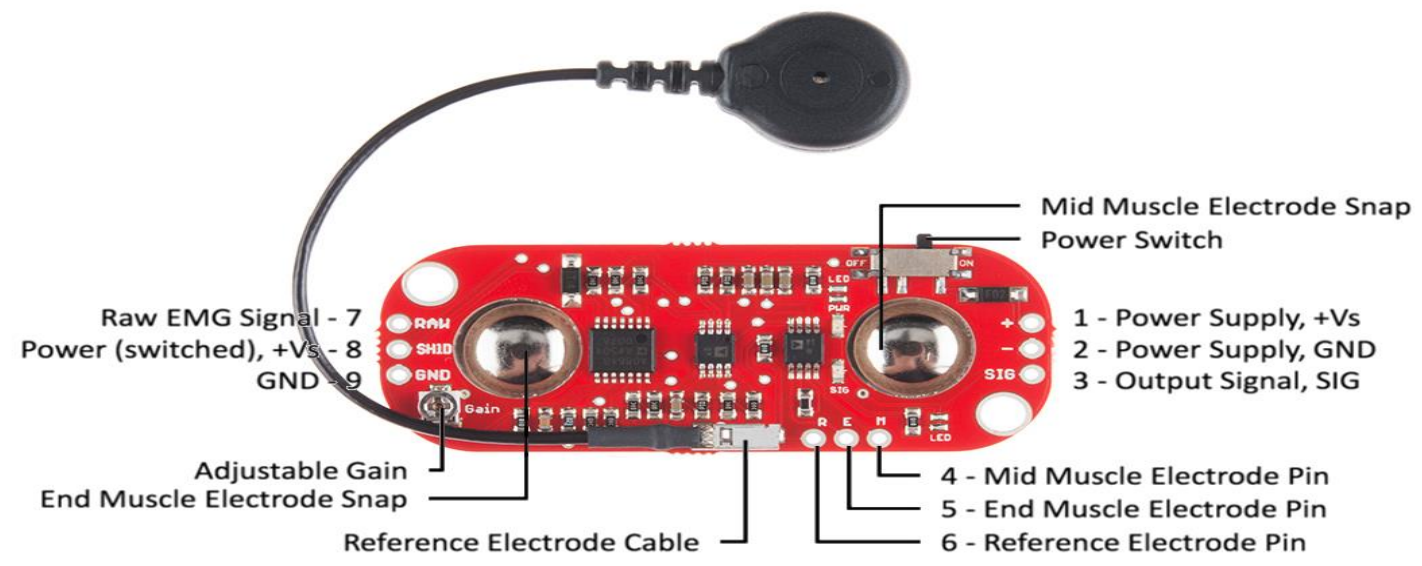

FIG. 1 MYOWARE MUSCLE SENSOR STRUCTURE [21].

Fortunately, the Myoware muscle sensor adopted in this project is specifically designed to be directly utilized with a microcontroller such as Arduino boards. Therefore, Myoware outputs are not only a raw EMG signal but rather it can produce an amplified, rectified and integrated signal. These outputs work well with the analog to digital convertor microcontrollers. Fig. 2 illustrates the primary differences between raw EMG signals and enhanced EMG signals [22].

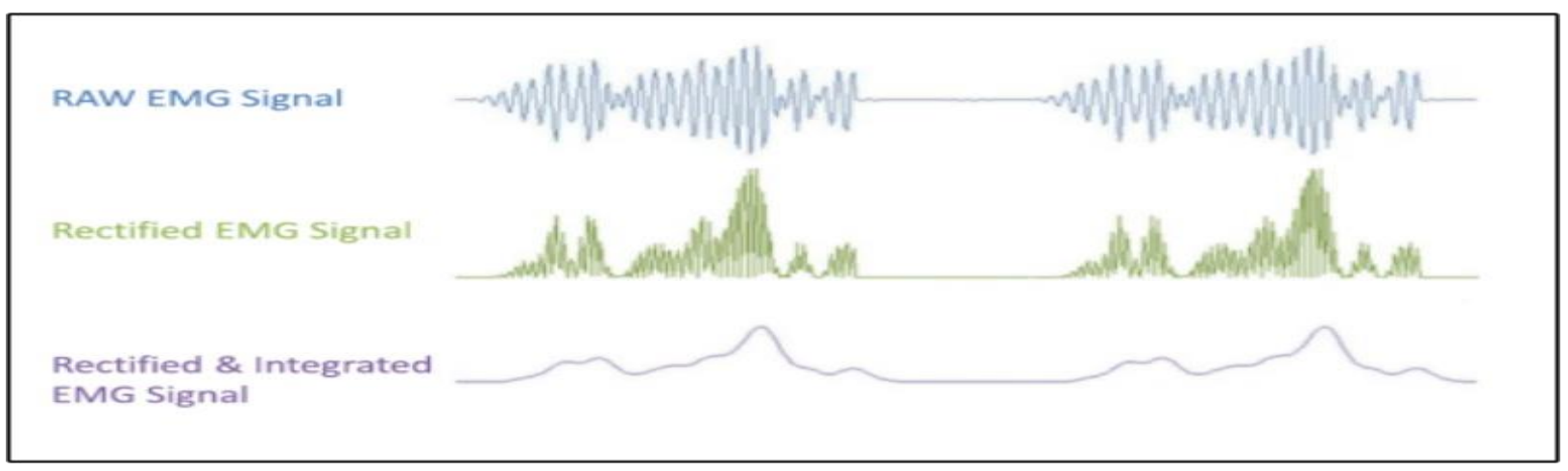

FIG. 2 TYPES OF EMG SIGNALS FROM MYOWARE MUSCLE SENSOR [23].

\section{B. The Block Diagram of the Proposed System}

In general, Fig. 3 presents the block diagram of the proposed system. It can be seen that the utilized microcontroller plays the main role in the proposed system. It collects the EMG signals from sensors and generates the required control signals.

The work flow of the proposed system in the two adopted case studies can be summarized as follows:

Step 1: Placing one channel surface EMG (sEMG) electrodes with muscle sensor at the middle of the desired muscle for recording raw EMG signals according to the following setup [24]:

- The first surface electrode must be located at the middle side of the target muscle and connected to the cable snap connector of Myoware muscle sensor. 
- The second surface electrode must be located at the one end of the target muscle and connected to the other cable snap connector of Myoware muscle sensor.

- The last surface electrode must be located on bony near the target muscle and connected to the cable snap connector of Myoware muscle sensor.

Step 2: Establishing wire connection between muscle sensor and microcontroller for managing these processed hand nerve signals to be adopted for many control applications.

Step 3:Structuring the proposed control algorithm for this study and converting it in a form of program to sense the movements of muscles and load the sensing program into the microcontroller, such as the Arduino UNO.

Step 4: Opening the serial plotter of Arduino IDE environment and then activating the desired muscle/muscles group to analyze the received hand nerve signals according to flag values and level of muscle flexing.

Step 5: Finally, the microcontroller makes a decision and provides the suitable EMG control commands based on the thresholding ranges, programmed flag values and level of muscle flexing in the proposed algorithm.

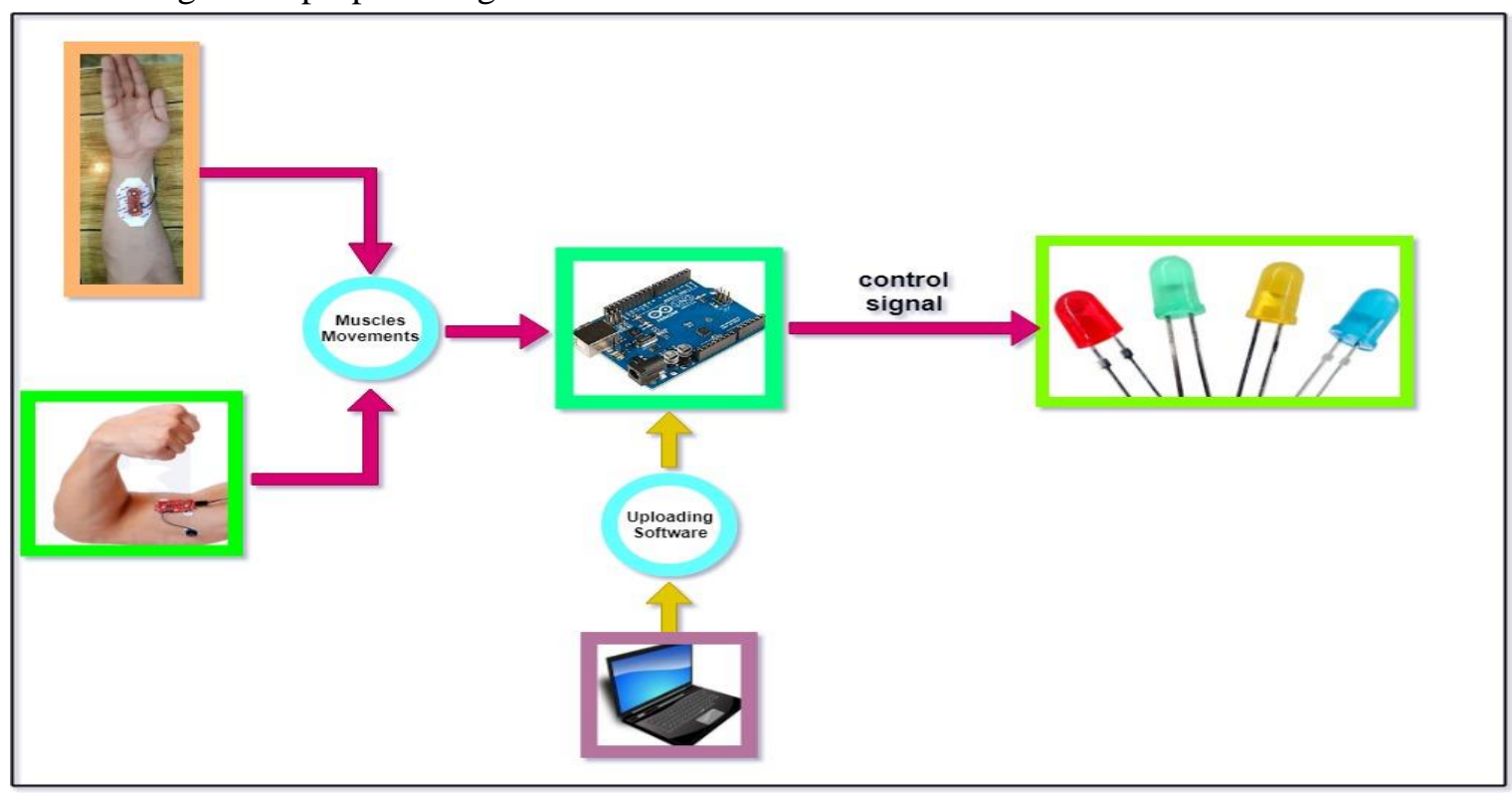

FIG. 3 BLOCK DIAGRAM OF THE PROPOSED SYSTEM

\section{The Proposed Algorithm}

In general, two case studies have been adopted in this work for tackling different types of paralyzing. The muscle movements, which have been adopted for generating the control signals in the first case study, are listed in Table II, while the muscle movements, which have been adopted for generating the control signals in the second case study, are listed in Table III. This proposed algorithm performs the required classification of upper limb motions according to the threshold ranges as shown in Table IV.

TABLE II. THE FIRST CASE STUDY IN DETAILS

\begin{tabular}{|c|l|}
\hline Control Commands & \multicolumn{1}{|c|}{ Action of muscles } \\
\hline Green & Ring finger muscle is actived softly only one time within the loop period \\
\hline Yellow & Ring finger muscle is actived softly only two times within the loop period \\
\hline Red & $\begin{array}{l}\text { Ring finger and middle finger muscles are actived hardly only one time within the loop } \\
\text { period }\end{array}$ \\
\hline Blue & $\begin{array}{l}\text { Ring finger and middle finger muscles are actived hardly only two times within the loop } \\
\text { period }\end{array}$ \\
\hline Off & All fingers muscles are relaxed \\
\hline
\end{tabular}


TABLE III. THE SECOND CASE STUDY IN DETAILS

\begin{tabular}{|c|l|}
\hline Control Commands & \multicolumn{1}{|c|}{ Action of muscle } \\
\hline Green & The biceps muscle is activated softly only one time within the loop period \\
\hline Yellow & The biceps muscle is activated softly only two times within the loop period \\
\hline Red & The biceps muscle is activated hardly only one time within the loop period \\
\hline Blue & The biceps muscle is activated hardly only two times within the loop period \\
\hline Off & Biceps muscle is relaxed \\
\hline
\end{tabular}

TABLE IV. THRESHOLDING RANGES OF EMG SIGNALS

\begin{tabular}{|l|l|l|}
\hline \multirow{2}{*}{ EMG signals } & \multicolumn{2}{|c|}{ Thresholding ranges } \\
\cline { 2 - 3 } & First testing & Second testing \\
\hline EMG signal 1 & $\mathrm{TH} 1=140 \rightarrow 480$ & $\mathrm{TH} 1=120 \rightarrow 450$ \\
\hline EMG signal 2 & $\mathrm{TH} 2>500$ & $\mathrm{TH} 2>480$ \\
\hline
\end{tabular}

This paper gives a high performance control system by structuring a high efficiency control algorithm with cheap hardware equipment. However, the proposed control algorithm is designed to control the operation and extinguishing of colored LEDs as a prototype that can be replaced by any device or moving object such as robot or wheelchair, according to the hand nerve signals of the upper limb amputees people. Fig. 4 shows the flowchart of the proposed algorithm.

The proposed algorithm goes through the following steps:

1. Initially, the sensing software declares flag $i$ as an integer equals to zero for controlling the loop of eight counts, this loop is repeated continuously.

2. Inside the loop

- The sensing software causes a delay of two seconds until the muscle sensor obtains the sense of hand nerve signals from the desired muscle/muscles group that is activated.

- Reading and displaying EMG signals on the serial plotter of the Arduino UNO for analyzing these signals based on opening serial monitor of the Arduino UNO and showing the digital values of EMG signals to make a decision according to the specified conditions inside the sensing program.

- Flags values $(Z, B, W)$ depend on how strong the muscle contraction is.

- The contraction of the desired muscle is activated smoothly for only one time within the assigned loop period, which will increase the value of the flag $Z$ by one.

- The contraction of the desired muscle is activated smoothly and only two times within the assigned loop period, which will increase the value of the flag $Z$ by two.

- The contraction of the desired muscle is activated strongly for only one time within the assigned loop period, which will increase the value of the flag $W$ by one.

- The contraction of the desired muscle is activated strongly for only two times within the assigned loop period, which will increase the value of the flag $W$ by two.

- Relaxing the desired muscle within the assigned loop period, which will increase the value of the flag $B$ by one at each relax.

3. Outside the loop

- After the period of the loop is completed, the classification process will be performed for generating the appropriate decision in the form of a control command to control the operation of four colored LEDs depending on the values of the flags $(Z, W$, and $B)$ and threshold ranges inside the program.

4. Finally, after producing the appropriate control command, this loop is repeated continuously for another action from hand gestures. 


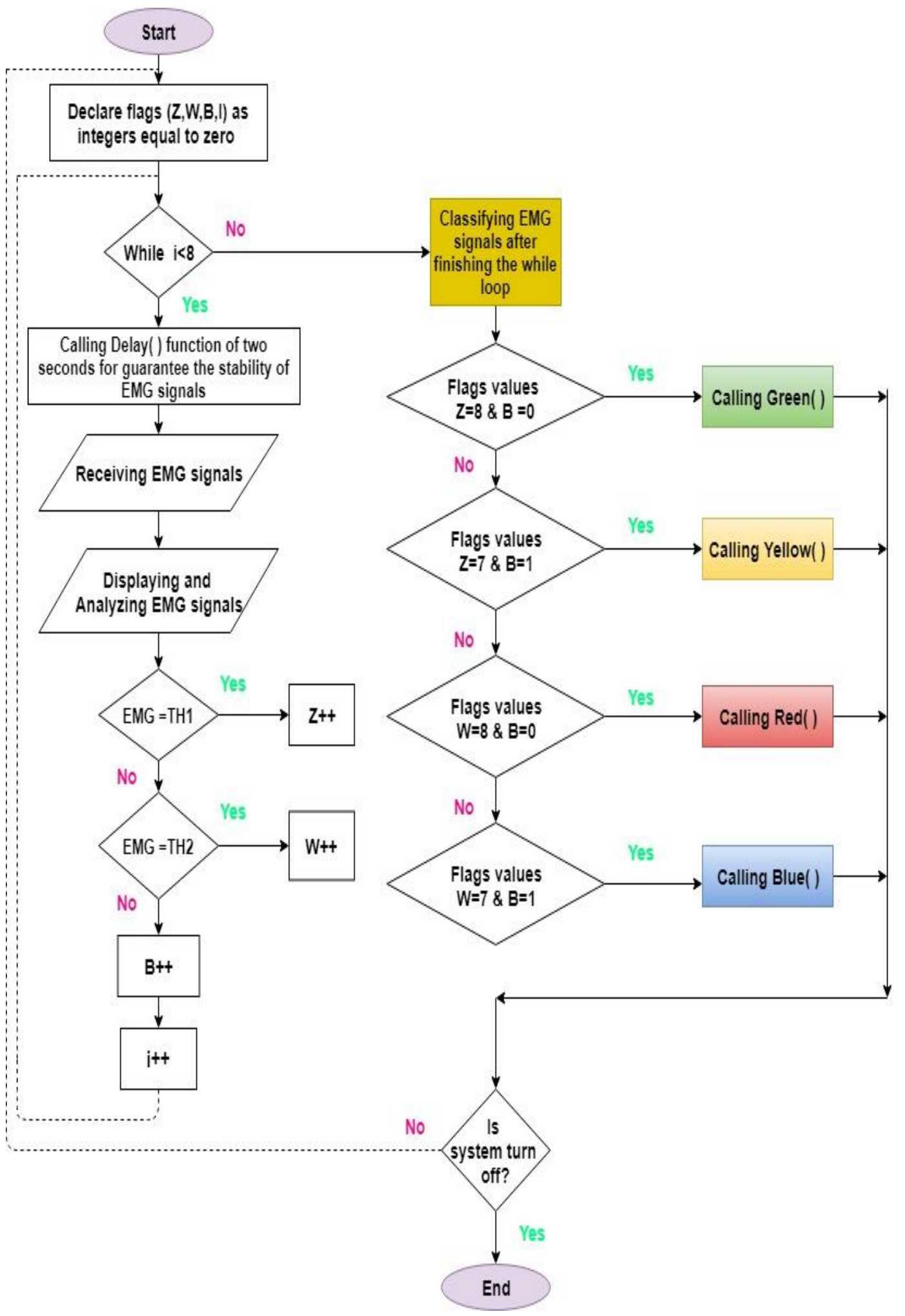

FIG. 4 FLOWCHART OF THE PROPOSED ALGORITHM

\section{Classification of Hand Motions}

Ten types of fingers and arm movements are exploited for classification criteria in this work for two case studies. The success rate of these classification criteria depends on the level of muscle flexing and the value of flags in the suggested control algorithm within the same loop period according to Table $\mathrm{V}$ for the first case study and Table VI for the second case study. 
TABLE V. CLASSIFICATION CRITERIA OF THE FIRST CASE STUDY

\begin{tabular}{|l|l|r|r|}
\hline Target muscle & Level of flexing & Flag value & Classification symbols \\
\hline Ring finger & Low (softly) & $\mathrm{Z}=1$ & $\mathrm{~F} 1$ \\
\hline Ring finger & Low (softly) & $\mathrm{Z}=2$ & $\mathrm{~F} 2$ \\
\hline Ring and middle fingers & High (hardly) & $\mathrm{Z}=1$ & $\mathrm{~F} 3$ \\
\hline Ring and middle fingers & High (hardly) & $\mathrm{Z}=2$ & $\mathrm{~F} 4$ \\
\hline All fingers relaxed & Relax & $\mathrm{Z}=0$ & $\mathrm{~F} 5$ \\
\hline
\end{tabular}

TABLE VI. CLASSIFICATION CRITERIA OF THE SECOND CASE STUDY

\begin{tabular}{|l|l|r|c|}
\hline Target muscle & Level of flexing & Flag value & Classification symbols \\
\hline Biceps muscle & Low (softly) & $\mathrm{W}=1$ & F6 \\
\hline Biceps muscle & Low (softly) & $\mathrm{W}=2$ & F7 \\
\hline Biceps muscle & High (hardly) & $\mathrm{W}=1$ & F8 \\
\hline Biceps muscle & High (hardly) & W=2 & F9 \\
\hline Biceps relaxed & Relax & W=0 & F10 \\
\hline
\end{tabular}

The accuracy of selecting the appropriate location of electromyography surface electrodes with the adopted muscle sensor is considered very paramount to acquire more robust and precise information about all upper limb actions to guarantee the validation of the classification criteria. As a useful summary, the number of movements possible for giving high classification rate is directly dependent on the location of the muscle sensor on the target muscles.

After acquisition and processing of EMG signals in the Myoware muscle sensor and classification of these signals according to the sensing program, which relies on the second structured control algorithm, the transmission of signals takes place through the connection of Arduino and MyoWare muscle sensor.These signals are received at the Arduino analog pins, which then convert the signal through programming and provide the digital output. The converted (digital) signals are used to control the operation of four colored LEDS in four basic colors, namely: GREEN, YELLOW, RED and BLUE as a prototype.

\section{EXPERIMENTATION AND TESTING RESULT}

The purpose of this work is to generate different control commands based on hand nerve signals that are read by the muscle sensor. These readings are sent to the Arduino UNO microcontroller for preprocessing procedure and forming the output control commands. These commands are used to control the operation of ON/OFF LEDs as a prototype that can be replaced by any other robot or moving objects. The first experiment adapts itself to record the average of classification accuracy of the ten hand movements in the two case studies of the amputees people by stabilizing the location of the muscle sensor in each case study with measurement of classification success rate of all discussed movements according to equation (1).

\section{correct classification attempts

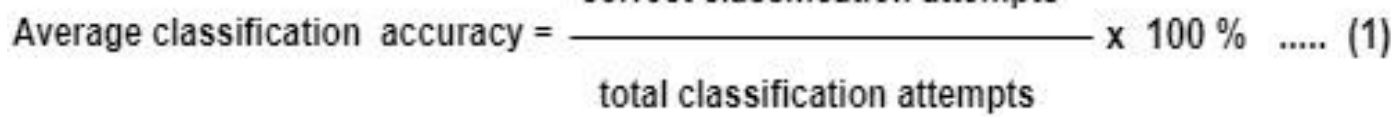

In this experiment, we take into account the first testing digital thresholding ranges that are listed in Table IV. The testing results of the first experiment are shown in Table VII and Table VIII for the first and the second case studies, respectively. In this experiment, the total average classification accuracy reached to $92.335 \%$ from the result of ten muscles movements that are listed in Table VII and Table VIII. 
TABLE VII. THE FIRST CASE STUDY TESTING RESULT(THE FIRST EXP)

\begin{tabular}{|l|l|l|}
\hline Classification Symbols & $\begin{array}{l}\text { Average } \\
\text { Classification } \\
\text { Accuracy }\end{array}$ & $\begin{array}{c}\text { Control } \\
\text { Command }\end{array}$ \\
\hline F1 & $100 \%$ & Green ON \\
\hline F2 & $88.2 \%$ & Yellow ON \\
\hline F3 & $88.2 \%$ & Red ON \\
\hline F4 & $82.3 \%$ & Blue ON \\
\hline F5 & $100 \%$ & OFF \\
\hline F1+F2+F3+F4+F5 & \multicolumn{2}{|c|}{$91.74 \%$} \\
\hline
\end{tabular}

TABLE VIII. THE SECOND CASE STUDY TESTING RESULT(THE FIRST EXP)

\begin{tabular}{|l|c|l|}
\hline Classification Symbols & $\begin{array}{c}\text { Average } \\
\text { Classification } \\
\text { Accuracy }\end{array}$ & $\begin{array}{c}\text { Control } \\
\text { Command }\end{array}$ \\
\hline F6 & $100 \%$ & Green ON \\
\hline F7 & $82.35 \%$ & Yellow ON \\
\hline F8 & $94.11 \%$ & Red ON \\
\hline F9 & $88.23 \%$ & Blue ON \\
\hline F10 & $100 \%$ & OFF \\
\hline F6+F7+F8+F9+F10 & \multicolumn{2}{|c|}{$92.93 \%$} \\
\hline
\end{tabular}

The second experiment employs itself to record the average of classification accuracy of the ten hand movements in the two case studies of the amputees people by stabilizing the location of the muscle sensor in each case study with measurement of classification success rate of all discussed actions according to equation (1).

In this experiment, we take into account the second testing digital thresholding ranges that are listed in Table IV. The testing results of the second experiment are shown in Table IX and Table X for the first and the second case studies, respectively. In this experiment, the total average classification accuracy reached to $95.285 \%$ from the result of ten muscles movements that are listed in Table IX and Table X.

TABLE IX. THE FIRST CASE STUDY TESTING RESULT(THE SECOND EXP)

\begin{tabular}{|l|l|l|}
\hline Classification symbols & $\begin{array}{l}\text { Average } \\
\text { classification } \\
\text { accuracy }\end{array}$ & $\begin{array}{c}\text { Control } \\
\text { Command }\end{array}$ \\
\hline F1 & $100 \%$ & Green ON \\
\hline F2 & $94.11 \%$ & Yellow ON \\
\hline F3 & $88.23 \%$ & Red ON \\
\hline F4 & $88.23 \%$ & Blue ON \\
\hline F5 & $100 \%$ & OFF \\
\hline F1+F2+F3+F4+F5 & \multicolumn{2}{|c|}{$94.11 \%$} \\
\hline
\end{tabular}

TABLE X. THE SECOND CASE STUDY TESTING RESULT(THE SECOND EXP)

\begin{tabular}{|l|c|l|}
\hline Classification Symbols & $\begin{array}{c}\text { Average } \\
\text { Classification } \\
\text { Accuracy }\end{array}$ & $\begin{array}{c}\text { Control } \\
\text { Command }\end{array}$ \\
\hline F6 & $100 \%$ & Green ON \\
\hline F7 & $94.11 \%$ & Yellow ON \\
\hline F8 & $94.11 \%$ & Red ON \\
\hline F9 & $94.11 \%$ & Blue ON \\
\hline F10 & $100 \%$ & OFF \\
\hline F6+F7+F8+F9+F10 & \multicolumn{2}{|c|}{$96.46 \%$} \\
\hline
\end{tabular}


In general, from the two experiments, this system can accomplish a total classification accuracy of $93.81 \%$ from ten muscles actions in each experiment with different thresholding ranges.

The success of the first muscle movement (F1) depends on the contracting of the ring finger muscle softly only once within the time of the specified loop duration with the adopted threshold ranges. The result of F1 (the first action in 1'st CS) is shown in Fig. 5 .

The success of the second muscle movement (F2) depends on the contracting of the ring finger muscle softly only twice within the time of the specified loop duration based on the considered threshold ranges. The result of F2 (the second action in 1'st CS) is shown in Fig. 6.

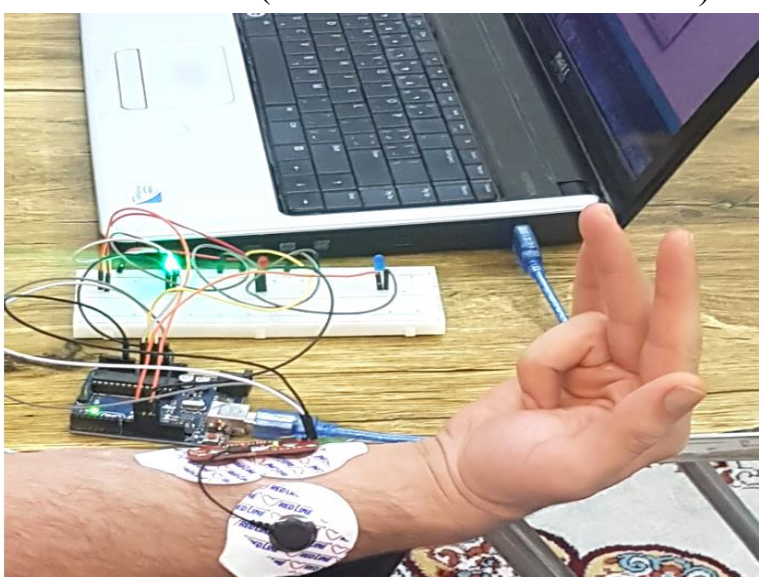

FIG. 5 RESULT OF F1 (Z=1)

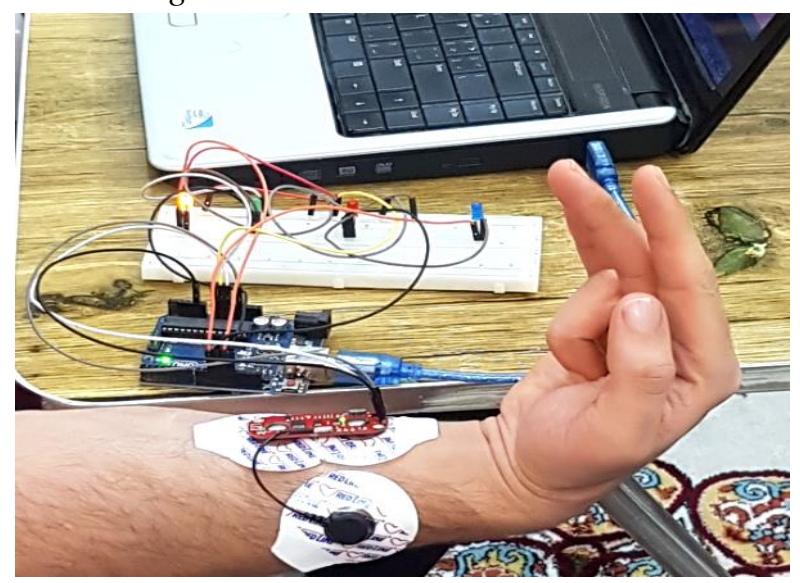

FIG. 6 RESULT OF F2 (Z=2)

The third muscle movement (F3) is successfully performed depending on the contracting of the ring, middle finger muscles strongly only one within the time of the specified loop duration, which is done based on the adopted thresholding ranges. The result of F3 (the third action in 1'st CS) is shown in Fig. 7.

The forth muscle movement (F4) is successfully performed depending on the contracting of the ring, middle finger muscles strongly only twice within the time of the specified loop duration based on the considered thresholding ranges. The result of F4 (the forth action in 1'st CS) is shown in Fig. 8.

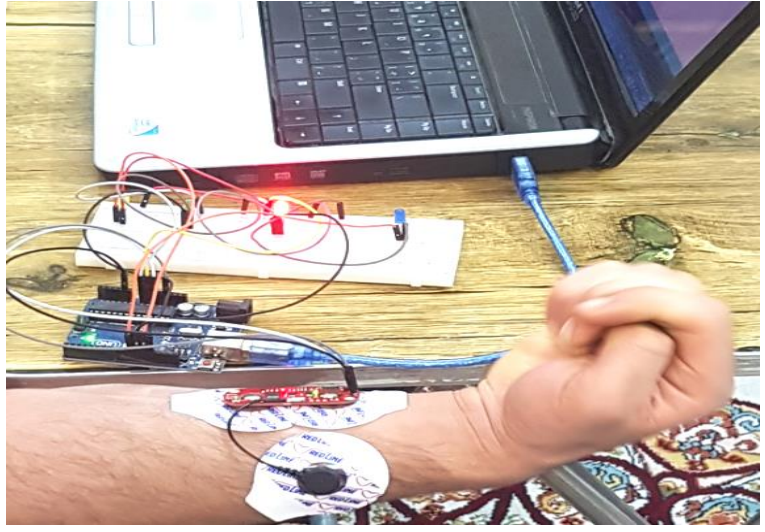

FIG. 7 RESULT OF F3 (Z=1)

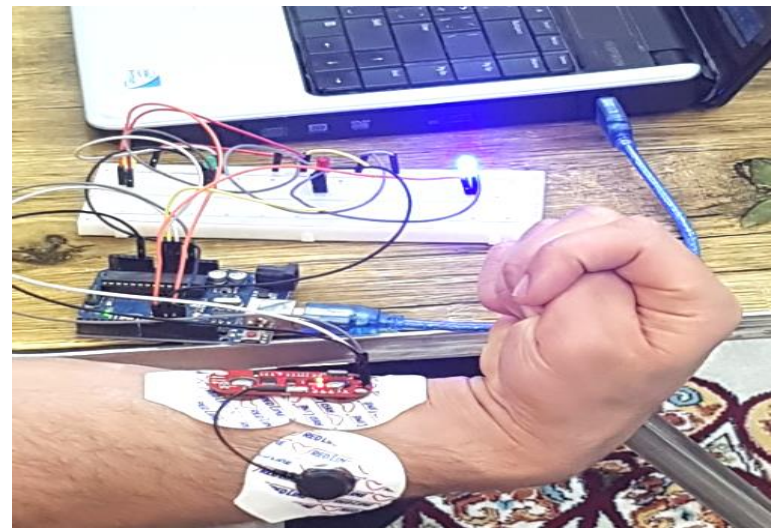

FIG. 8 RESULT OF F4 (Z=2)

The fifth muscle movement (F5) is successfully performed when all fingers muscles are relaxed within the time of the specified loop. The result of F5 (the fifth action in 1'st CS) is shown in Fig. 9.

The sixth muscle movement (F6) uses the biceps muscle (case study two) with strongly contracting only once within the time of the specified loop duration based on threshold ranges. The result of F6 (the first action in 2'nd CS) is shown in Fig. 10. 


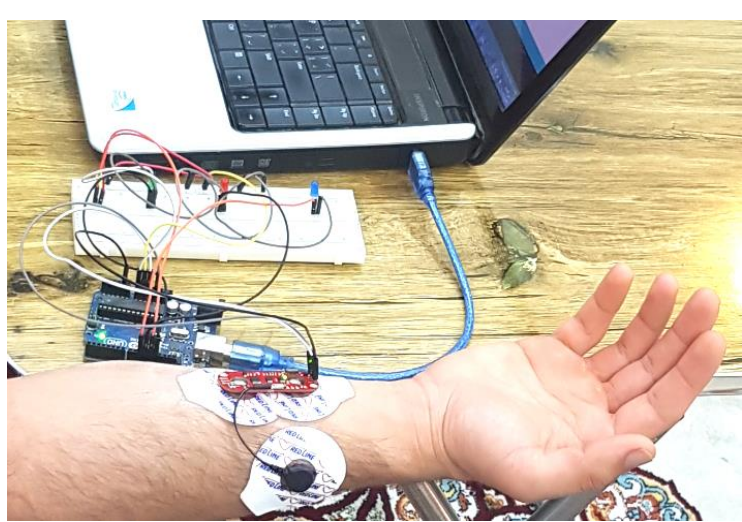

FIG. 9 RESULT OF F5

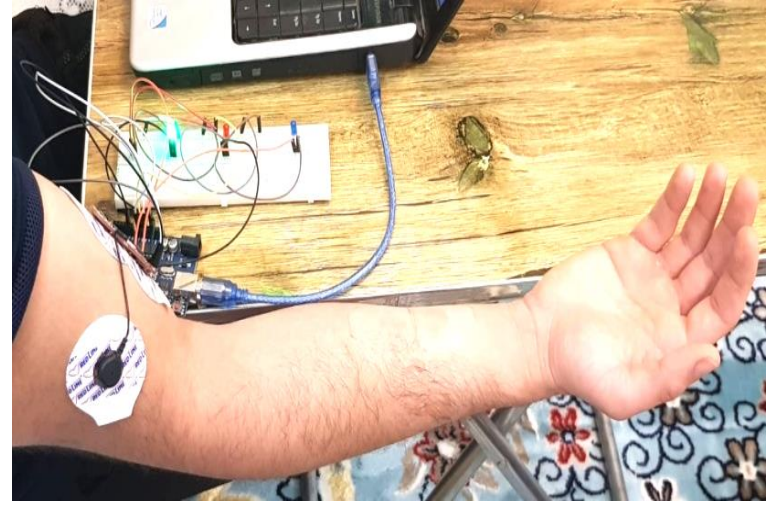

FIG. 10 RESULT OF F6 (W=1)

The seventh muscle movement (F7) adopts the biceps muscle with softly flexing only twice within the time of the specified loop duration at the threshold ranges, as shown in Fig. 11.

The eighth muscle movement (F8) employes the biceps muscle with very hardly contracting only once within the time of the specified loop duration according to the threshold ranges, as shown in Fig. 12.

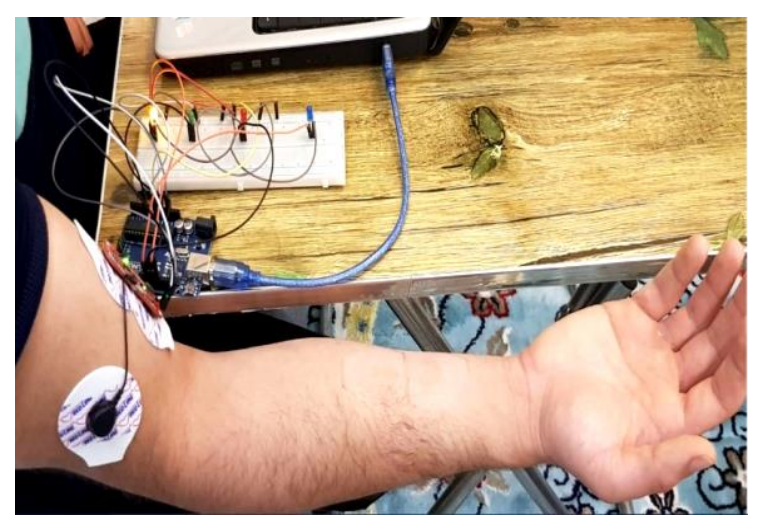

FIG. 11 RESULT OF F7 (W=2)

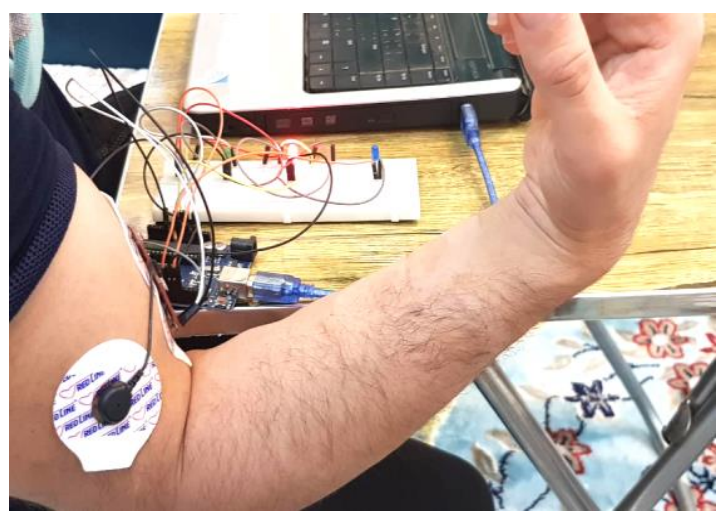

FIG. 12 RESULT OF F8 (W=1)

The ninth muscle movement (F9) adopts the biceps muscle with very hardly flexing only twice within the time of the specified loop duration based on the specified thresholding ranges. The result of F9 (the forth action in 2'nd CS) is shown in Fig. 13.

The tenth muscle movement (F10) is successly performed when the biceps muscle is relaxed within the time of the specified loop duration. The result of F10 (the fifth action in 2'nd CS) is shown in Fig. 14.

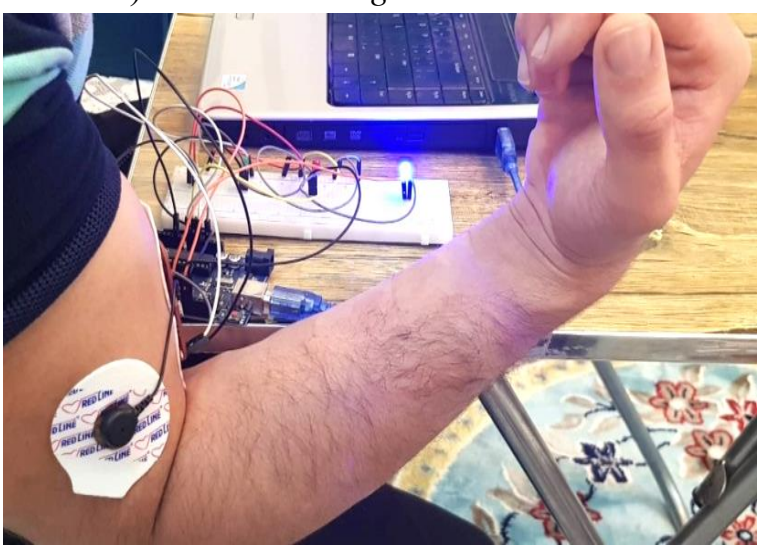

FIG. 13 RESULT OF F9 (W=2)

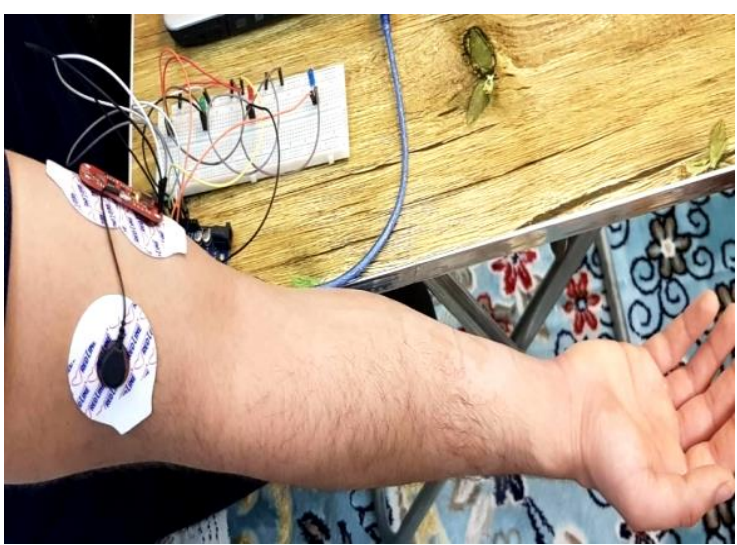

FIG. 14 RESULT OF F10 


\section{CONCLUSION}

In this paper, a control system was proposed to assist people with upper limb movement problems based on hand-nerve signals as control signals. These signals were appeared in the form of control commands from the Arduino UNO microcontroller according to the proposed intelligent control algorithm that classifies ten actions from hand muscles. This system is tested over two suggested case studies to simulate different hand amputation cases. The obtained results proved that the classification accuracy is almost $93.81 \%$ from two experiments, each with ten muscles movements but with different thresholding ranges. The proposed algorithm gave more flexibility to the system with high controlling performance by adapting precise muscles movements according to the suggested flag values of the sensing program and the level of flexing the target muscles. The main feature of the proposed system, which should be taken into consideration, is the low cost through the use of only one channel surface EMG (sEMG) electrodes with one muscle senor as cheap hardware equipment. As a useful summary, the proposed system was represented as a prototype that can be modified in the future to allow any aspect in the patient's dwelling to be controlled. This could include an ordinary wheelchair controlling, home appliances or personal devices controlling, which makes it eligible and adequate to be used as an assistant to the bilateral amputation people.

\section{REFERENCES}

[1] Azab, A. M., Onsy, A., \& El-Mahlawy, M. H. (2015). Design and Development of a Low Cost Prosthetic Arm Control System Based on sEMG Signal. Volume 3: Biomedical and Biotechnology Engineering. Doi: 10.1115/imece2015-51006.

[2] Cicek, Ecem, Zeynep Ozer, and Selma Oncel. "New Trends and Issues Proceedings on Humanities and Social Sciences." (2017).

[3] Lisi, G., Belluco, P., Cattaneo, D., \& Gini, G. (2011). From the Classification of EMG Signals to the Development of a New Lower Arm Prosthesis. IFAC Proceedings Volumes, 44(1), 6493-6498.

[4] Phinyomark, A., Limsakul, C., \& Phukpattaranont, P. (2011). A Review of Control Methods for Electric Power Wheelchairs Based on Electromyography Signals with Special Emphasis on Pattern Recognition. IETE Technical Review, 28(4), 316.doi:10.4103/0256-4602.83552

[5] Tsenov, G., et al. Neural Networks for Online Classification of Handand Finger Movements Using Surface EMG signals. in 8th Seminaron Neural Network Applications in Electrical Engineering(NEUREL). 2006.

[6] You, K.-J., Rhee, K.-W., \& Shin, H.-C. (2010). Finger Motion Decoding Using EMG Signals Corresponding Various Arm Postures. Experimental Neurobiology, 19(1), 54. doi:10.5607/en.2010.19.1.54

[7] Lisi, G., Belluco, P., Cattaneo, D., \& Gini, G. (2011). From the Classification of EMG Signals to the Development of a New Lower Arm Prosthesis. IFAC Proceedings Volumes, 44(1), 6493-6498. doi:10.3182/20110828-6-it-1002.02097

[8] Al-Timemy, A., et al., "Finger Movements Classification for theDexterous Control of Upper Limb Prosthesis Using EMG Signals,"Advances in Autonomous Robotics, no., pp. 434-435, 2012.

[9] Khushaba, R. N., Kodagoda, S., Takruri, M., \& Dissanayake, G. (2012). Toward improved control of prosthetic fingers using surface electromyogram (EMG) signals. Expert Systems with Applications, 39(12), 10731-10738. doi:10.1016/j.eswa.2012.02.192

[10] Anam, K., Khushaba, R. N., \& Al-Jumaily, A. (2013). Two-channel surface electromyography for individual and combined finger movements. 2013 35th Annual International Conference of the IEEE Engineering in Medicine and Biology Society (EMBC).

[11] Shin, S., Tafreshi, R., \& Langari, R. (2014). A performance comparison of hand motion EMG classification. 2nd Middle East Conference on Biomedical Engineering. doi:10.1109/mecbme.2014.6783276

[12] Puttasakul, T., Sangworasil, M., \& Matsuura, T. (2015). Realization of robust real time robotic arm control system based on EMG signal. 2015 8th Biomedical Engineering International Conference (BMEiCON).

[13] Gregory Luppescu , Michael Lowney \& Raj Shah . (2016). Classification of hand gestures using surface electromyography signals for upper-limb amputees.

[14] Phukpattaranont, P., Thongpanja, S., Anam, K., Al-Jumaily, A., \& Limsakul, C. (2018). Evaluation of feature extraction techniques and classifiers for finger movement recognition using surface electromyography signal. Medical \& Biological Engineering \& Computing. doi:10.1007/s11517-018$1857-$ 
[15] Kyuwan Choi, Sato, M., \& Koike, Y. (2006). A new, human-centered wheelchair system controlled by the EMG signal. The 2006 IEEE International Joint Conference on Neural Network Proceedings. doi:10.1109/ijenn.2006.247118

[16] Reaz, M. B. I., Hussain, M. S., \& Mohd-Yasin, F. (2006). Techniques of EMG signal analysis: detection, processing, classification and applications. Biological Procedures Online, 8(1), 11-35. doi:10.1251/bpo115

[17] Jang, G., Kim, J., Lee, S., \& Choi, Y. (2016). EMG-Based Continuous Control Scheme With Simple Classifier for Electric-Powered Wheelchair. IEEE Transactions on Industrial Electronics, 63(6), 36953705. doi:10.1109/tie.2016.2522385

[18] Lisi, G., Belluco, P., Cattaneo, D., \& Gini, G. (2011). From the Classification of EMG Signals to the Development of a New Lower Arm Prosthesis. IFAC Proceedings Volumes, 44(1), 6493-6498. doi:10.3182/20110828-6-it-1002.02097

[19] Cañizares, A., Pazos, J., \& Benítez, D. (2017, November). On the use of 3D printing technology towards the development of a low-cost robotic prosthetic arm. In 2017 IEEE International Autumn Meeting on Power, Electronics and Computing (ROPEC) (pp. 1-6). IEEE.

[20] Budiharto, Widodo. "LOW COST PROSTHETIC HAND BASED ON 3-LEAD MUSCLE/ELECTROMYOGRAPHY SENSOR AND 1 CHANNEL EEG."

[21] https://www.pololu.com/product/2732.

[22 ] Nguyen, Ngoc. "Developing A Low-cost Myoelectric Prosthetic Hand." (2018).

[23] Budiharto, Widodo. "LOW COST PROSTHETIC HAND BASED ON 3-LEAD MUSCLE/ELECTROMYOGRAPHY SENSOR AND 1 CHANNEL EEG."

[24] Salama, M., \& Bakr, A. (2016). Six Prosthetic Arm Movements Using Electromyogram Signals: A Prototype. 2016 UKSim-AMSS 18th International Conference on Computer Modelling and Simulation (UKSim). doi:10.1109/uksim.2016.19. 